\title{
Merkel cell carcinoma in the community setting: a case report
}

\author{
Cameron M. Callaghan, MD, MPH, MS'1, Rumpa Amornmarn, MD² \\ ${ }^{1}$ Department of Radiation Oncology, Carver College of Medicine, The University of lowa, lowa City, IA; \\ ${ }^{2}$ Department of Radiation Oncology, Osceola Cancer Center, Kissimmee, FL, USA
}

Merkel cell carcinoma (MCC) is a rare neuroendocrine tumor of the skin initially believed to arise from the Merkel cells. In the community setting a general radiation oncologist may only encounter this pathology in a handful of cases over the course of their career. Due to the low incidence of this malignancy, few prospective randomized controlled trials have ever been conducted and therefore guidelines are based on relatively lower levels of evidence upon which the clinical recommendations are made. We discuss the case of a female in her 90s presenting with a classic MCC primary lesion, as well as satellite lesions proximal to both the primary and the draining regional lymph nodes with no evidence of nodal involvement. Here we discuss the presentation, management, treatment planning, underlying pathology, results and sequelae of treatment. We also review new treatment modalities, and the most current staging systems and guidelines.

Keywords: Merkel cell carcinoma, Metastatic, Recurrent, Electron and photon treatment, Multiple primaries

\section{Introduction}

Merkel cell carcinoma (MCC) is a relatively recently described clinical entity with the first known publication describing the pathology occurring in 1974 [1]. The title 'Merkel cell' has subsequently been shown to be somewhat of a misnomer, as the malignant cells most likely do not arise from mature Merkel cells themselves, but possibly from either cutaneous progenitor cells [2], or even more recent evidence suggests a possible origin in the early B-cell lineage [3]. Epidemiologic data reveals that it is a relatively rare but increasingly prevalent malignancy $[4,5]$, whose risk factors include age, immune compromise, UV exposure, and most recently, a viral association with a member of the polyomaviridae family now dubbed 'Merkel cell polyomavirus' (MCPyV) discovered in 2008

\section{$[6,7]$.}

Both the incidence and mortality of MCC rose markedly from 1986 to 2011 according to a large scale study of the Surveillance Epidemiology and End Results registry ( $n=$ $5,211)[8]$. Prognosis is poor and has generally been associated with nodal involvement, stage at presentation, and immune status [6]. Surgical resection with adjuvant radiation therapy has good control rates for locoregional disease but recurrence is common and a poor prognostic sign. Metastatic disease has historically been treated with platinum-based chemotherapeutic regimens but this approach has never been shown to improve overall survival $[9,10]$. It should be noted that the level of evidence for clinical recommendations pertaining to management of MCC (and especially recurrent MCC) is relatively low, as to date only one prospective

Received 09 October 2017, Revised 08 January 2018, Accepted 15 January 2018.

Correspondence: Cameron M. Callaghan, MD, MPH, MS, Department of Radiation Oncology, Carver College of Medicine, The University of lowa, lowa City, IA, USA. Tel: +1-407-619-0492, Fax: +1-319-356-1530, E-mail: Cameron.Callaghan@ucf.edu

(c) This is an Open Access article distributed under the terms of the Creative Commons Attribution Non-Commercial License (http://creativecommons.org/ licenses/by-nc/4.0/) which permits unrestricted non-commercial use, distribution, and reproduction in any medium, provided the original work is properly cited.

www.e-roj.org 


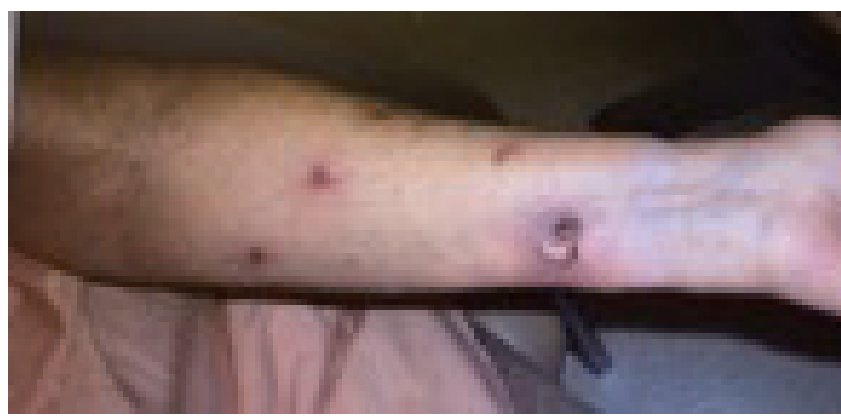

Fig. 1. Initial presentation status-post shave biopsies (black arrow indicates primary lesion) on March 2008.

randomized controlled trial of MCC has ever been reportedwhich had to conclude prematurely due to changes in standard of care [11].

\section{Case Report}

We report a case of a female in her 90s with past medical history of bilateral breast cancer status-post ( $s / p$ ) bilateral mastectomy approximately 60 years prior to presentation, non-small cell lung cancer (NSCLC) s/p lobectomy 7 years prior to presentation with no other past medical history, first noting a skin lesion on her distal left volar forearm 9-12 months prior to presenting to Dermatology in early March of 2008. Since first noticing the primary skin lesion it had grown, become painful, and three new smaller lesions had appeared, now involving the medial bicep, proximal volar forearm, and medial volar forearm of her left upper extremity (LUE). Dermatology performed shave biopsies of all lesions. Pathology report noted positive margins in all biopsies demonstrating "...nodular densely cellular neoplasm in the dermis composed of sheets of small round cells with large hyperchromatic nuclei and scant cytoplasm...", which stained positive for CK20 (in a perinuclear dot-like pattern), synaptophysin, and negative for S-100, pancytokeratin, and thyroid transcription factor-1 (TF1). Pathologists' diagnosis was a neuroendocrine carcinoma consistent with MCC and she was referred to radiation oncology.

Physical exam demonstrated multiple reddish lesions 0.5-3.0 $\mathrm{cm}$ in diameter with the largest on the distal left volar forearm with ulceration (Fig. 1). Patient declined any further workup and initial treatment plan was with palliative intent with no future plans of chemotherapy, lymph node biopsy, or PET scan per the patients wishes. Radiation treatment plan initially included 6,000 cGy in 20 fractions (fx) to the primary and 3,000 $\mathrm{cGy} / 10 \mathrm{fx}$ to the three distant sites of disease using 6-MV electron beam prescribed to the $90 \%$ line with $0.5 \mathrm{~cm}$ bolus and $1 \mathrm{~cm}$ margins around gross tumor volume.

After the initial $10 \mathrm{fx}$ of this course the three distant sites had completely resolved by clinical inspection. However, disease was still evident at the primary site and three new lesions had appeared in the left lateral forearm, the left medial forearm, and the left upper arm. All three new sites of disease were independent of the original lesions, representing either new metastases or lesions that had extended along dermal lymphatics far from the radiation fields and not representing 'marginal misses'. The three new distant lesions were treated with the same regimen as the previous distant sites of disease with 3,000 cGy/10 fx of 6-MV electron beam, while the primary was continued on its original treatment plan to a total of 6,000 cGy/20 fx which were completed at the end of April 2008.

On follow-up in early May of 2008 both the primary and previous distant sites of disease had completely resolved per clinical inspection but three new $0.5-1.0 \mathrm{~cm}$ lesions had appeared on the left upper arm; one on the medial aspect, on the ventral aspect and one on the lateral aspect (Fig. 2A). All
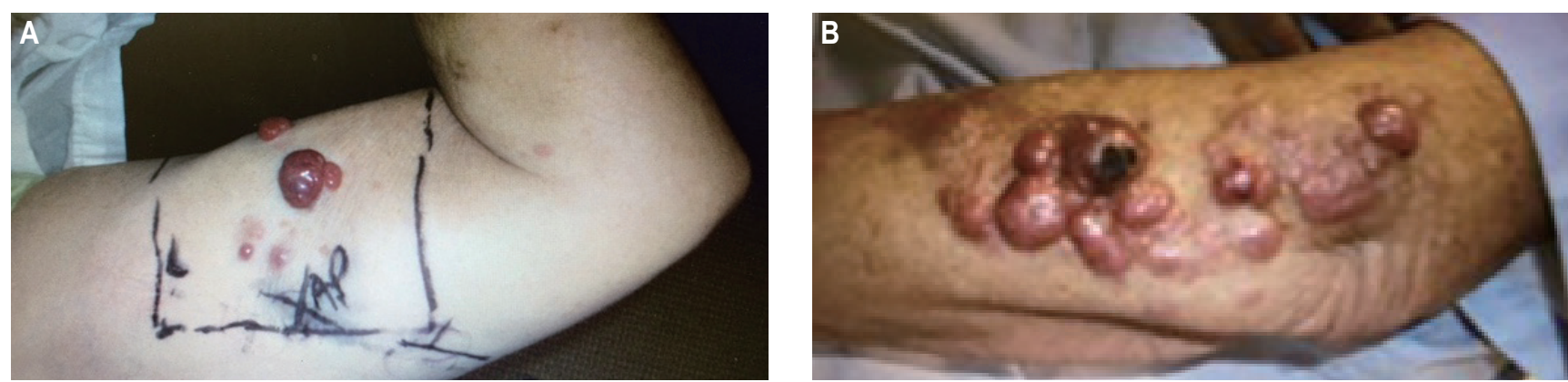

Fig. 2. The distant sites of disease. (A) Left upper arm recurrence (May 2008). (B) Recurrence on lateral aspect of left forearm (October 2008). 
A
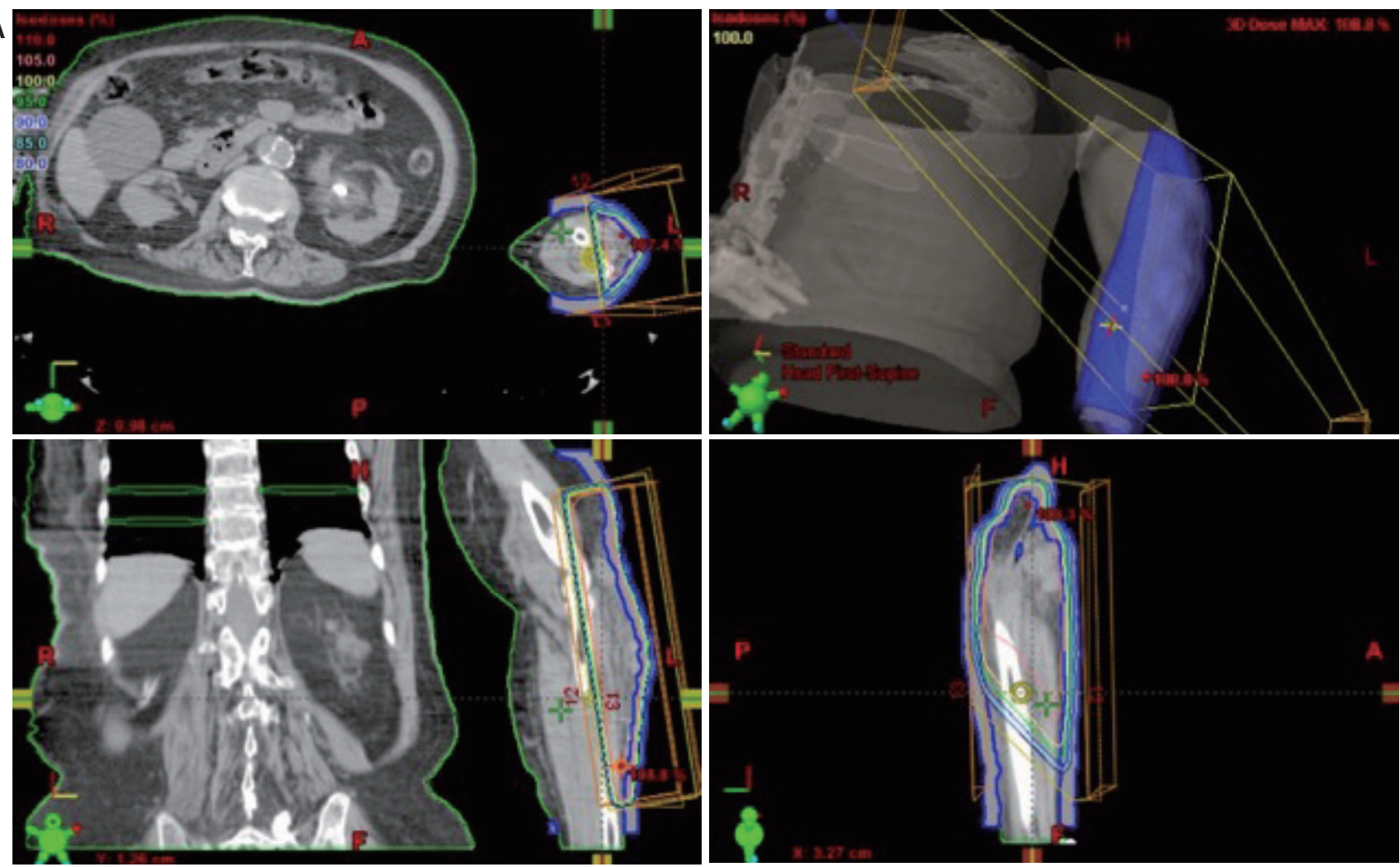

B
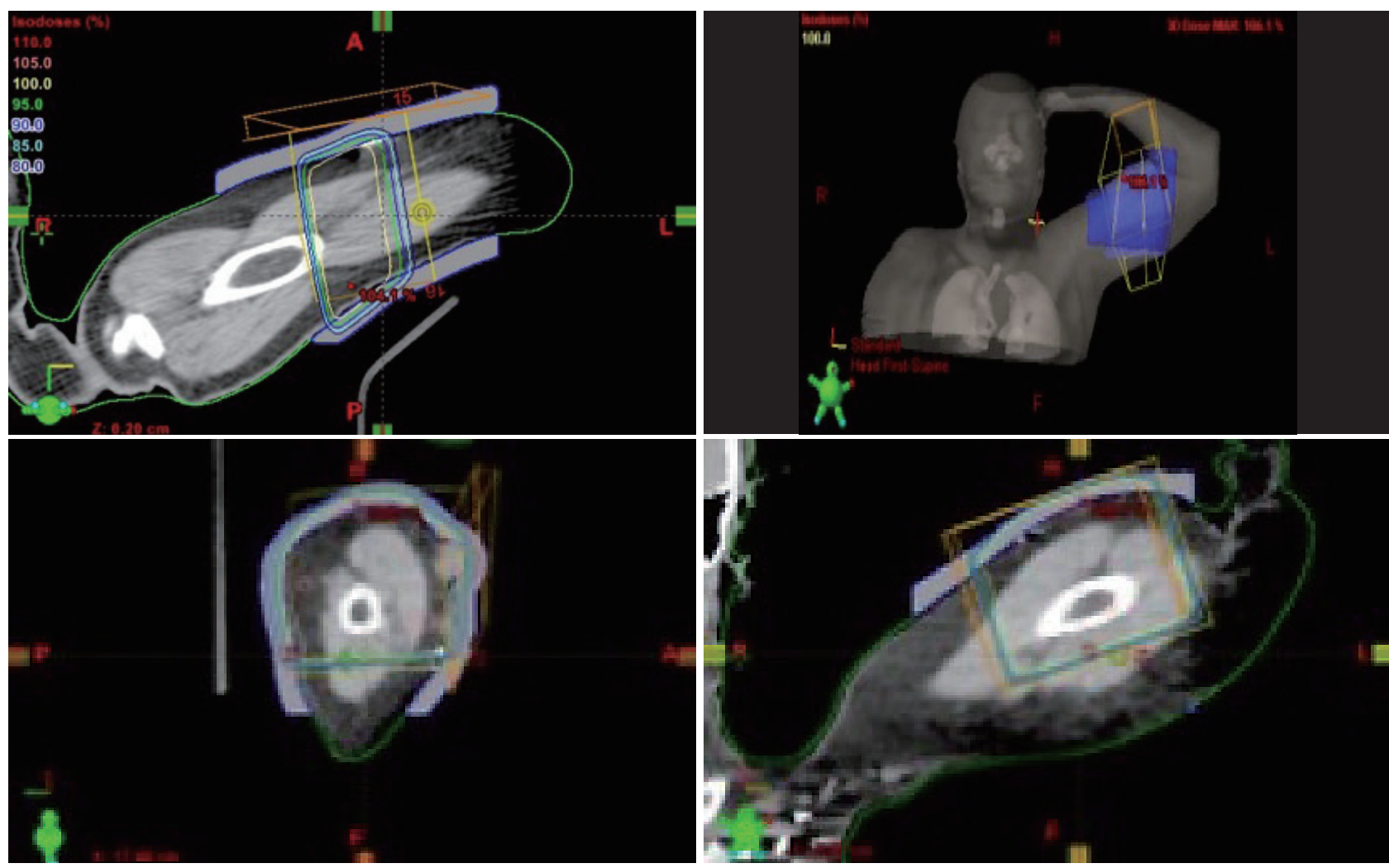

Fig. 3. (A) Three-dimensional conformal radiation therapy 6-MV photon beams with simple anteroposterior (AP) and posteroanterior (PA) portals treatment planning of left forearm (October 2008). (B) Treatment planning of left upper arm (October 2008). 

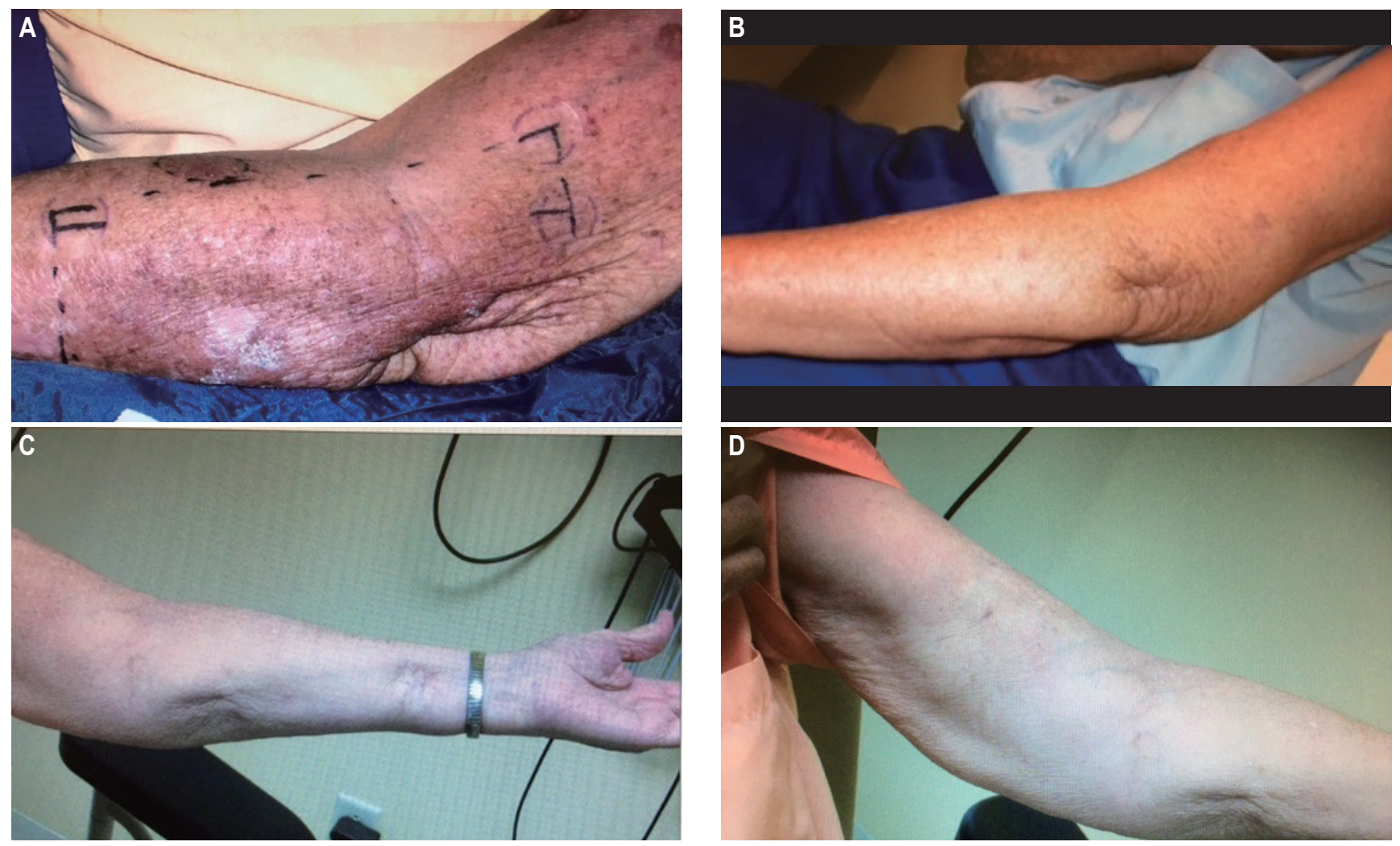

Fig. 4. (A) Complete resolution of lateral left forearm recurrence from Fig. 2B (November 2008). (B) Clinical remission of recurrent disease from Fig. 2B (May 2009). (C) No evidence of disease at 1-year follow-up from recurrence in Fig. 2B (November 2009). (D) Complete clinical resolution of recurrent disease from Fig. 2A in May 2008 and again in October 2008 (November 2009).

three new sites of disease were again found in locations distant from all previous lesions, representing either new metastases or extensions along dermal lymphatics far from previous radiation fields. All new lesions were treated with 2,000 cGy/5 fx of 6-MV electrons in early to May of 2008 with complete clinical resolution on follow-up.

Patient was then lost to follow-up until she presented again in early October of 2008 with multiple recurrences of 2-3 $\mathrm{cm}$ reddish nodular lesions on the LUE with the largest patch measuring $7 \mathrm{~cm} \times 12 \mathrm{~cm}$ while the previous lesion in the distal left forearm had completely regressed. Recurrent sites of disease were again in new locations distant of all previous sites, and presented on the lateral aspect of the left forearm (Fig. 2B), dorsal aspect of the left forearm, and on the left upper inner arm. The lesions on the lateral aspect of the left forearm were treated with 5,100 cGy/17 fx of 6-MV photon beam via 3D-CRT simple opposed AP and PA portals with the clinical target volume (CTV) defined using CT simulation and skin wire markings (Fig. 3A). The other lesions on the left forearm were treated with 4,500 cGy/15 fx of 6-MV electron beam while the lesions on the left upper inner arm were treated with 3,000 cGy/10 fx of 6-MV photon beam followed by a 1,500 cGy/5 fx boost using 6-MV electron beam (Fig. 3B). These courses started in early October 2008 and concluded in mid-November of that year with complete clinical resolution of the lesions (Fig. 4A).

Follow-up in January of 2009 patient was feeling much better, was experiencing no more pain and only slight tenderness to touch. On physical exam, she was noted to have complete regression of her lesions with some scarring at the original tumor sites and edema of the subcutaneous tissue of the inner aspect of the left forearm. On follow-up in May 2009 there was no evidence of disease (Fig. 4B). Patient had noticed some swelling in her left arm and on physical exam was found to have some discoloration of the skin in the treated area with some edema of the inner aspect of the left arm.

At follow-up in November of 2009 patient denied any symptoms or pain but noticed an increase in size of a reddish lesion on her chest and a new skin lesion on her left forearm. Physical exam revealed diminution of the left arm edema, and 
a 5-cm scar at the previous surgical site on the left forearm. Other previously treated lesions had completely regressed without scar formation (Fig. 4C and 4D). There was one $0.5 \mathrm{~cm}$ raised reddish lesion on the dorsal surface of the left forearm 5 $\mathrm{cm}$ above the wrist. Clinical impression of the chest lesion was basal cell carcinoma (BCC) or squamous cell carcinoma (SCC), and the new left forearm lesion was presumed to represent a MCC recurrence. Patient was referred to dermatology for biopsy of the left chest wall lesion and active surveillance of left forearm lesion with radiotherapy if it increased in size.

On follow-up in late April of 2010 the lesion on the left chest wall had been excised with pathology demonstrating $\mathrm{BCC}$, and two skin lesions on the left arm had been cauterized by dermatology. On physical exam, there was no edema in the left arm, well healed scar with no signs of recurrence on the left chest wall, and two dry scabs on the left forearm and left upper arm from cauterizations performed by dermatology. After this point, the patient lived for an additional 3 years with no evidence of recurrence, dying of non-malignant causes approximately 5 years after initial presentation.

\section{Discussion}

This is a classic case of MCC in terms of histopathology, and clinical presentation and course. Patient is elderly, with classic lesions occurring on the sun-exposed upper extremities (Fig. 1). By American Joint Committee on Cancer (AJCC) 8th edition staging system [12] this would be staged as T2NOpM1a at presentation, yielding a prognostic stage IV (Table 1). After wide local excision, a course of radiation therapy to a total dose of 56-60 Gy in 28-30 fx of photons and/or electron beam to the primary tumor would be indicated given the microscopically positive margins and per the most recent National Comprehensive Cancer Network (NCCN) Guidelines [13]. In this case a combination treatment using both photon and electron beam therapy was broken up into anatomic segments to decrease the potential morbidity of delivering 60 Gy to the entire LUE. Despite extensive spread representing either skin metastases or spread along dermal lymphatics, systemic therapy was not initiated as immune checkpoint inhibitors were not available at the time, and systemic platinum-based chemotherapy was declined due to the patient's age and preferences. No clinical trials were both available and logistically feasible for the patient at the time of treatment.

The current literature does not offer much guidance regarding management of recurrences in MCC and individualized treatment on a case-by-case basis is often left to the clinician's personal experience and judgement. Recent studies have examined the possible use of the anthracycline amrubicin as a systemic salvage therapy [14] and, due to the role of anaplastic Iymphoma kinase (ALK) in MCC, the potential of the ALK inhibitor crizotinib may warrant further investigation [15]. A murine model study demonstrated that the histone deacetylase inhibitor vorinostat can stimulate the re-expression of human leukocyte antigen class-I on the surface of MCC cells, therefore potentially decreasing their ability to evade immune-surveillance [16]. Vorinostat has also been approved for use in the treatment of cutaneous T-cell Iymphoma.

Histopathologically MCC is CK20 and synaptophysin positive while being negative for S-100, pancytokeratin, and TF-1 (Table 2). It is unknown if this case represents carcinogenesis via UV radiation exposure or $\mathrm{MCPyV}$. There are, as of yet, no validated recommendations for alternate treatment regimens based on viral status, and it is not yet routinely tested; although a recent study suggests that MCPyV-negative cases of MCC carry a worse prognosis both in terms of disease progression and MCC-specific mortality [17]. MCPyV testing was not performed in this patient as it was not available at the time of treatment. That same study suggested that cases of MCPyV-negative MCC could be identified with high sensitivity and specificity using a CM2B4 antibody. Most recent NCCN Guidelines (version 1.2018) states that testing for oncoprotein antibodies for MCPyV "may be considered" at presentation [13].

Where this case diverges from the typical MCC case is the past history of other malignancies and the duration of clinical disease-free survival, especially given the prognostic stage at presentation. This patient a past history of breast cancer ( $s / p$ definitive treatment in the distant past with no evidence of disease), NSCLC (s/p lobectomy 7 years prior to presentation without further intervention or sequelae), and was discovered to have concurrent BCC of the skin during the course of re-treatment. An association had been noted between breast cancer, chronic lymphocytic leukemia (CLL), and concurrent non-melanotic skin cancer with MCC [18]; however, this study had a fairly small sample size $(n=17)$. In 2010, Danish study ( $n=185$ ) confirmed the associations of SCC and CLL with MCC, but did not find associations with breast cancers or NSCLC [19]. A recent study of NSCLC patients demonstrated that infection rates with MCPyV were Iow $(4.7 \%-17.9 \%)$, but when present was significantly correlated with prognosis-suggesting MCPyV may play a role in some cases of both MCC and NSCLC [20]. Now that larger databases 

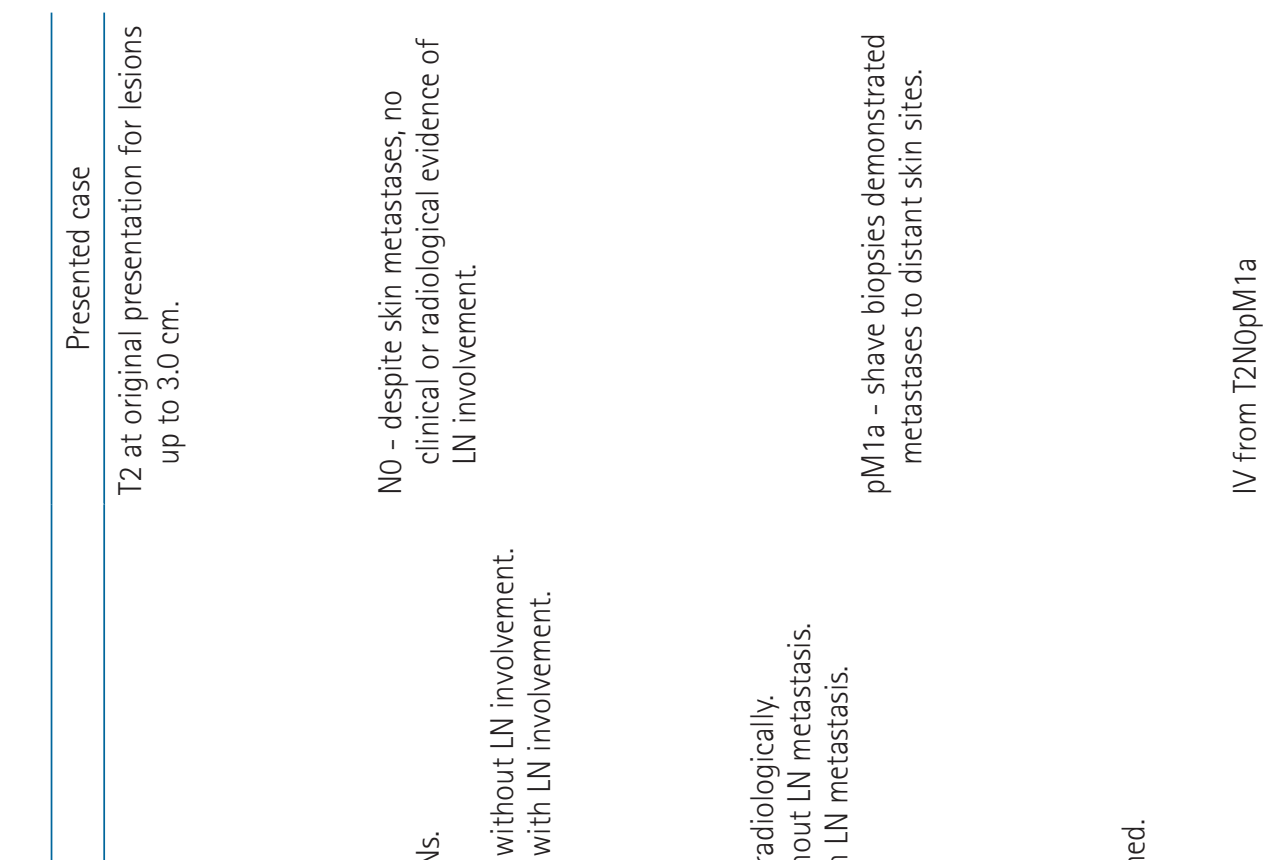

夏

춘

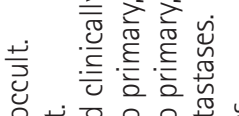

ठั.

入

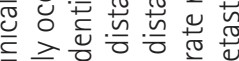

U.

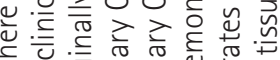

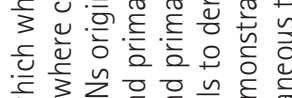

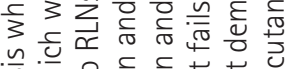

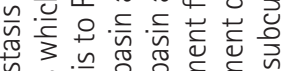

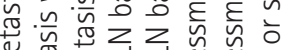

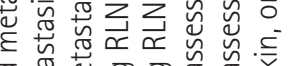

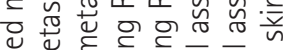

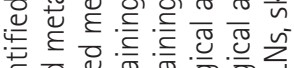

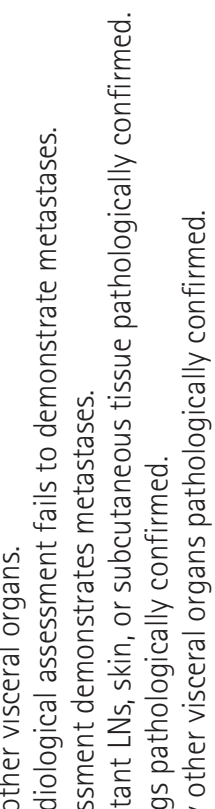

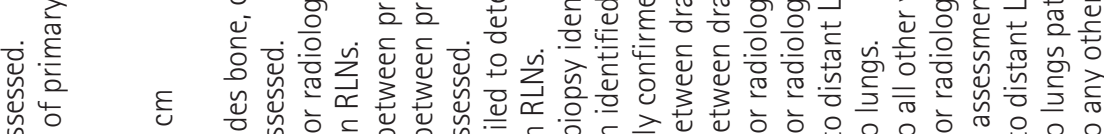

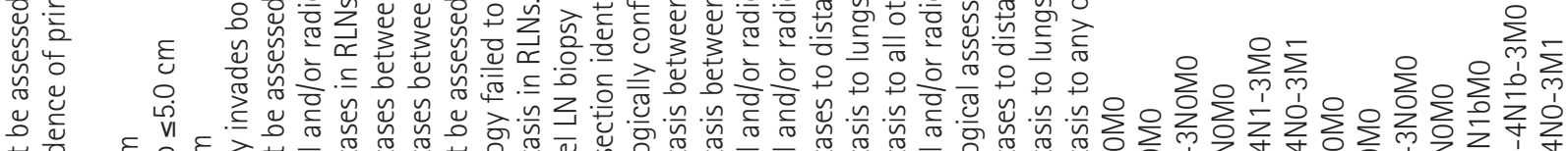

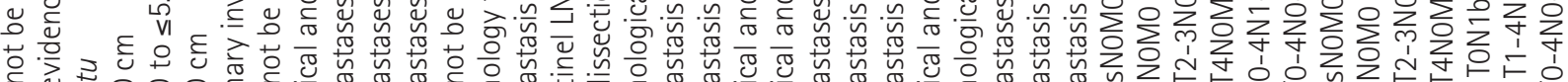

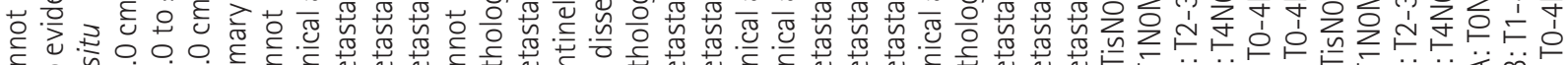

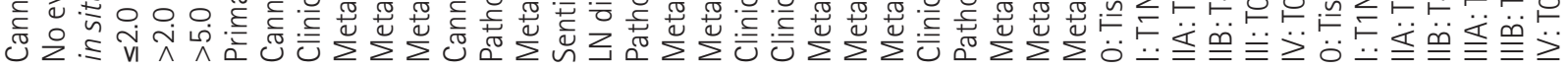

\section{$\frac{\frac{\pi}{n}}{\frac{\pi}{n}}$}

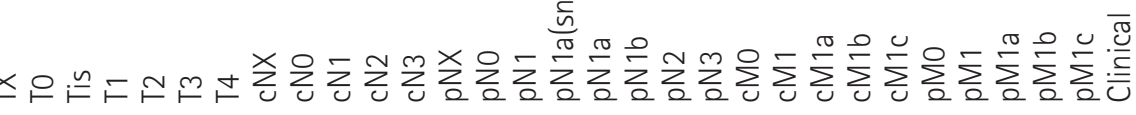

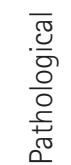




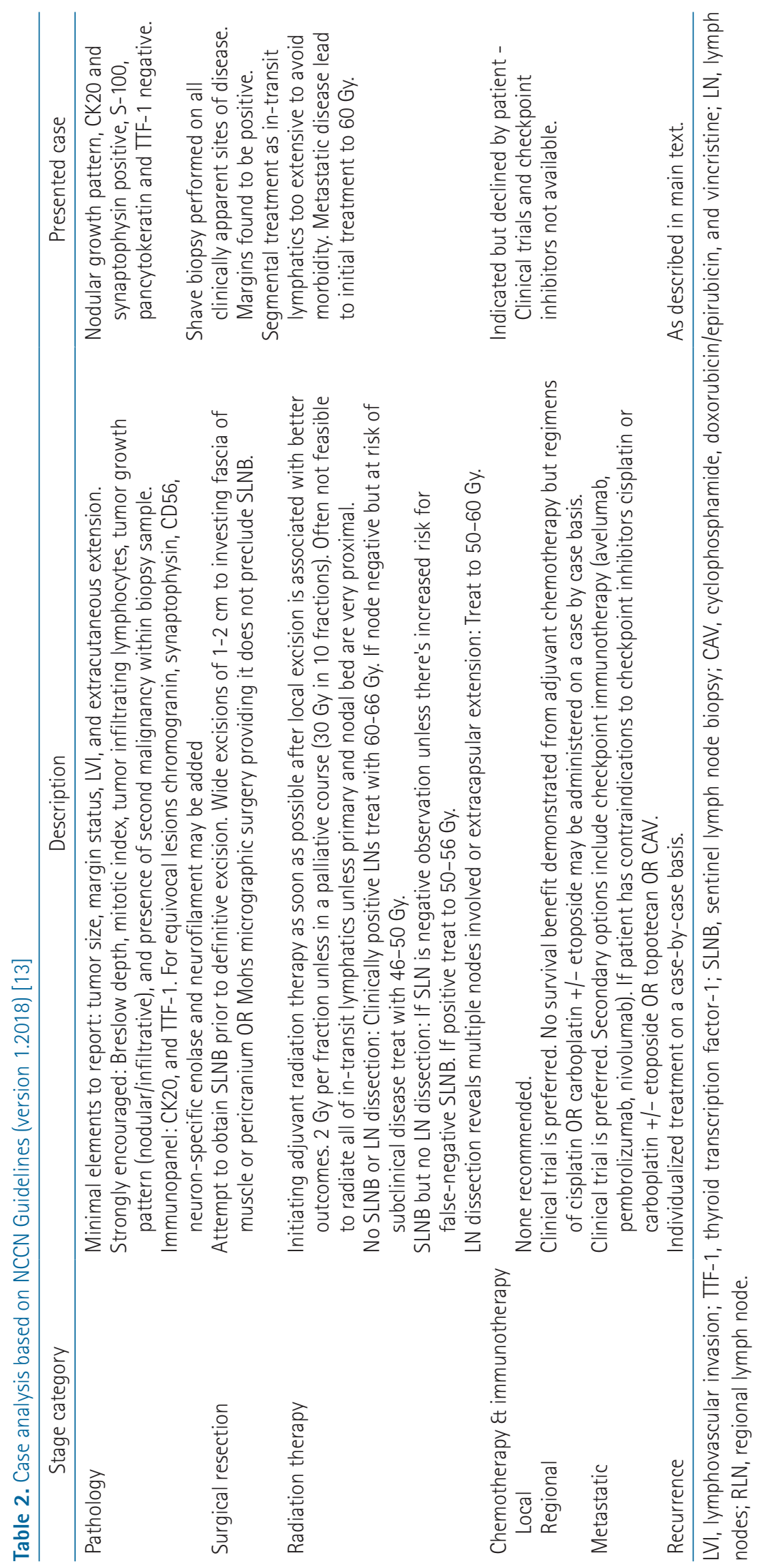


of MCC exist it may be illustrative to investigate further.

In conclusion, while the pathogenesis of MCC is being elucidated and new treatment modalities offer exciting avenues for therapy, MCC remains an aggressively recurrent malignancy with poor prognosis and increasing incidence. More large scale prospective randomized controlled trials are needed in order to improve the quality of evidence for clinical guidelines-especially in the context of recurrence and for the use of checkpoint inhibitors.

\section{Conflict of Interest}

No potential conflict of interest relevant to this article was reported.

\section{Acknowledgments}

First and foremost, the authors would like to thank the patient in this case for adding to the scientific body of knowledge and to all the previous investigators in this subject area for their contributions.

\section{References}

1. Toker C. Trabecular carcinoma of the skin. Arch Dermatol 1972;105:107-10.

2. Tilling T, Moll I. Which are the cells of origin in Merkel cell carcinoma? J Skin Cancer 2012;2012:680410.

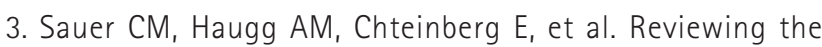
current evidence supporting early B-cells as the cellular origin of Merkel cell carcinoma. Crit Rev Oncol Hematol 2017;116:99-105.

4. Amaral T, Leiter U, Garbe C. Merkel cell carcinoma: epidemiology, pathogenesis, diagnosis and therapy. Rev Endocr Metab Disord 2017;18:517-32.

5. Santamaria-Barria JA, Boland GM, Yeap BY, Nardi V, DiasSantagata D, Cusack JC. Merkel cell carcinoma: 30-year experience from a single institution. Ann Surg Oncol 2013;20:1365-73.

6. Banks PD, Sandhu S, Gyorki DE, Johnston ML, Rischin D. Recent insights and advances in the management of Merkel cell carcinoma. J Oncol Pract 2016;12:637-46.

7. Schadendorf D, Lebbe C, Zur Hausen A, et al. Merkel cell carcinoma: Epidemiology, prognosis, therapy and unmet medical needs. Eur J Cancer 2017;71:53-69.

8. Fitzgerald TL, Dennis $S$, Kachare SD, Vohra NA, Wong JH, Zervos EE. Dramatic increase in the incidence and mortality from Merkel cell carcinoma in the United States. Am Surg 2015;81:802-6.

9. Garneski KM, Nghiem P. Merkel cell carcinoma adjuvant therapy: current data support radiation but not chemotherapy. J Am Acad Dermatol 2007;57:166-9.

10. Tai P. Merkel cell cancer: update on biology and treatment. Curr Opin Oncol 2008;20:196-200.

11. Jouary $T$, Leyral C, Dreno B, et al. Adjuvant prophylactic regional radiotherapy versus observation in stage I Merkel cell carcinoma: a multicentric prospective randomized study. Ann Oncol 2012;23:1074-80.

12. Amin $M B$, Greene $F L$, Edge $S B$, et al. AJCC cancer staging manual. 8th ed. Cham, Switzerland: Springer International Publishing; 2017.

13. National Comprehensive Cancer Network. NCCN Clinical Practice Guidelines in Oncology: Merkel cell carcinoma 1.2018 [Internet]. Fort Washington, PA: National Comprehensive Cancer Network; c2018 [cited 2018 Mar 1]. Available from: https://www.nccn.org/professionals/physician_gls/default. aspx.

14. Sakaida $E$, Ebata $T$, Iwasawa $S$, et al. Potential activity of amrubicin as a salvage therapy for Merkel cell carcinoma. Intern Med 2017;56:567-70.

15. Cao S, Nambudiri VE. Anaplastic Iymphoma kinase in cutaneous malignancies. Cancers (Basel) 2017:9:123.

16. Ritter $C$, Fan $K_{1}$ Paschen $A$, et al. Epigenetic priming restores the HLA class-I antigen processing machinery expression in Merkel cell carcinoma. Sci Rep 2017;7:2290.

17. Moshiri AS, Doumani R, Yelistratova $L$, et al. Polyomavirusnegative Merkel cell carcinoma: a more aggressive subtype based on analysis of 282 cases using multimodal tumor virus detection. J Invest Dermatol 2017;137:819-27.

18. Brenner B, Sulkes A, Rakowsky E, et al. Second neoplasms in patients with Merkel cell carcinoma. Cancer 2001;91:1358-62.

19. Kaae J, Hansen AV, Biggar RJ, et al. Merkel cell carcinoma: incidence, mortality, and risk of other cancers. J Natl Cancer Inst 2010;102:793-801.

20. Kim GJ, Lee JH, Lee DH. Clinical and prognostic significance of Merkel cell polyomavirus in nonsmall cell lung cancer. Medicine (Baltimore) 2017;96:e5413. 